\title{
Quality of Reaching and Postural Control in Young Preterm Infants Is Related to Neuromotor Outcome at 6 Years
}

\author{
BJØRG FALLANG, INGVIL ØIEN, ELISABET HELLEM, OLA DIDRIK SAUGSTAD, AND \\ MIJNA HADDERS-ALGRA
}

Oslo University College, Health Sciences, Physiotherapy Programme, 0130 Oslo, Norway [B.F., I.Ø., E.H.], Department of Pediatric Research, The National Hospital, University of Oslo, 0027 Oslo, Norway [O.D.S.], University Hospital Groningen, Department of Neurology, 9713 GZ Groningen, The Netherlands [M.H.-A.]

\begin{abstract}
A substantial proportion of the "apparently normal" preterm infants exhibit minor and moderate dysfunctions in neuromotor outcome as they grow older. Birth characteristics, minor abnormalities on the neonatal ultrasound scan of the brain, and motor milestones have only limited value in the early detection of these children. The aim of the present study was to investigate whether nonoptimal reaching and relatively immobile postural behavior at an early age are associated with dysfunctional neuromotor and behavioural development at school age. The preterm children and full-term children of the present follow-up study participated in a previous study on the characteristics of reaching kinematics and the kinetics of posture at 4 and 6 mo corrected age. At the age of $6 \mathrm{y}$, the children were re-assessed by means of the Touwen neurologic assessment, the Movement $\mathrm{ABC}$, and the Child Behavior Check List. The results demonstrated that in preterm children without cerebral palsy, a lack of successful reaching at 4 mo and a nonoptimal quality of reaching at 6 mo are related to the development of a complex form of minor neurologic dysfunction (MND) and fine manipulative disability at $6 \mathrm{y}$. Thus, these early signs indicate the presence of clinically significant
\end{abstract}

\section{ABSTRACT}

brain dysfunction. A relatively immobile postural behavior at 4 mo was associated with simple MND, coordination problems, and at 6 mo with a worse score on the Movement $\mathrm{ABC}$ and internalizing behavior. This suggests that a relatively immobile postural behavior points to a mild form of brain dysfunction. (Pediatr Res 58: 347-353, 2005)

Abbreviations
CBCL, Child Behavior Checklist
COP, center of pressure
CP, cerebral palsy
FT, full-term
GM, general movement
HR, high-risk
LR, low-risk
MND, minor neurological dysfunction
MU, movement unit
PT, preterm
ROP, retinopathy of prematurity

A substantial proportion of "apparently neurologically normal" preterm infants do exhibit minor and moderate dysfunctions in neuromotor outcome as they grow older (1). Yet, these dysfunctions, which may seriously interfere with daily living activities, are not generally detected before school age. We therefore need to increase our knowledge of risk factors and patterns of early neuromotor development that relate to impaired motor outcome at school age. So far, the prospective

Received March 25, 2004; accepted November 16, 2004.

Correspondence: Bjørg Fallang, Oslo University College, Health Sciences, Physiotherapy Programme, Postbox 4, St. Olavs Place, 0130 Oslo, Norway; e-mail: bjorg.fallang@hf.hio.no

This study was supported by The Norwegian Foundation for Health and Rehabilitation, Oslo, Norway.

DOI: 10.1203/01.PDR.0000170898.60160.09 value of birth characteristics and minor abnormalities on the neonatal ultrasound scan of the brain have been inconsistent for later motor impairments in this preterm group $(1,2)$.

Prediction of motor impairments at school age on the basis of the assessment of motor milestones at an early age is hampered by the presence of many false negatives $(3,4)$. Prediction based on the quality of early motor behavior may be better. Recent work by Hadders-Algra et al. $(5,6)$ indicated that an abnormal quality of GM, which had been present until about 4 mo corrected age, was related to MND at early and later school age. A nonoptimal quality of specific motor functions, such as reaching and grasping at 9 mo, has also been associated with reduced quality of hand motor performance at $2.6 \mathrm{y}$ (7).

Preterm infants often show an abnormal quality of postural behavior (8), which has been addressed in terms of transient 
dystonia (9) or faulty muscle power regulation (10). But the significance of this specific postural behavior for the later development of preterm infants is not clear. Sommerfelt et al. (11) reported that transient dystonia that was observed at least once between 4 and 18 mo in preterm infants who were not later diagnosed as having cerebral palsy $(\mathrm{CP})$ was associated with a nonsignificant increase in neuromotor problems at $5 \mathrm{y}$. Samsom et al. (12), who included infants with serious lesions of the brain, found that muscle dysregulation at $3 \mathrm{mo}$ and dysfunctional postural control at 12 mo were the best predictors for a dysfunctional neurodevelopmental outcome at $7 \mathrm{y}$ of age. But de Vries and de Groot (7), who re-examined a selected low-risk sample of preterm infants, were unable to confirm a relationship between muscle dysregulation at $18 \mathrm{wk}$ and motor performance at $2.6 \mathrm{y}$.

We recently carried out a study as to how young preterm infants who were not later diagnosed as having $\mathrm{CP}$ organize goal-directed reaching and the accompanying postural adjustments $(13,14)$. The infants were studied at the corrected ages of 4 and 6 mo in supine position by means of kinematic recordings of reaching movements toward a toy. A forceplate on which the infant was lying recorded the postural adjustments, i.e. the movements of the total-body COP during the reach. Two major results with respect to reaching behavior were reported. First, we found that at 4 mo, low-risk preterm infants more often had an advanced reaching performance compared with full-term and high-risk preterm infants (13). The low-risk infants showed reaching movements with less MU, reaches during which the first $\mathrm{MU}$ - the so-called transport unitcovered a greater proportion of the reaching movement, and they showed reaches with a shorter total duration than the full-term and high-risk infants. MU are submovements of the reaching movement and are determined with the help of peaks in the velocity profile of the reaching hand. The better reaching performance of the low-risk infants was related to a better developmental outcome up to the age of $12 \mathrm{mo}$. Second, we found that at $6 \mathrm{mo}$, high-risk preterms significantly more often showed nonoptimal reaching movements than the full-term and low-risk preterm infants, but nonoptimal reaching was not related to performance on the Bayley scales at 6 and $12 \mathrm{mo}$. Nonoptimal reaching was defined on the basis of behavior on the compound variable "kinematic movement quality," which consisted of a combination of the variables peak velocity and number of MU (see also "Methods"). The main finding of the postural behavior was that the total-body COP in preterm infants was nearly motionless during reaching, whereas fullterm infants showed a mobile total-body COP. This difference between preterm and full-term infants was present both at 4 and 6 mo corrected age. In the low-risk preterm group, reduced postural motility was related to better reaching movements and to a normal quality of GM at 4 mo. We concluded that the relatively immobile postural behavior seemed to be an adequate strategy at an early age, but that it might be an early marker of dysfunctional development (14).

The aim of the present study was to investigate whether parameters of nonoptimal reaching and reduced COP behavior at an early age are associated with dysfunctional neuromotor and behavioral development at school age. The following questions were addressed: Are deviations in the kinematics of reaching in preterm children at an early age related to neurologic, motor and behavioral outcome at $6 \mathrm{y}$ ? Is the reduced total-body COP of preterm infants associated with dysfunctions in neurologic, motor, and behavioral outcome at $6 \mathrm{y}$ ? We also addressed the question of whether the presence of abnormal GM at 4 mo (5) was related to worse performance on neurologic, motor, or behavioral assessments at $6 \mathrm{y}$.

\section{METHODS}

Subjects. The subjects of the present project had participated in a previous study on characteristics of reaching and postural control during early infancy (13-15). Seventy-four Caucasian preterm infants had been enrolled [out of 88 eligible infants, see (12)]. The infants had been admitted to one of the three hospitals covering the city of Oslo (Ullevaal, Aker) and the nearest county (Akershus) during the period 1994 to 1996. Gestational age at birth was based on the date of the last menstrual period and confirmed by ultrasound measurement during early pregnancy. The primary inclusion criteria were a birthweight below $1750 \mathrm{~g}$ and a mother speaking either a Scandinavian language or English. Infants with a serious illness at 36 wk postmenstrual age or with cystic periventricular leukomalacia or intracranial haemorrhages extending into the parenchyma were excluded. Also excluded were children who at follow-up showed CP. The infants were allocated into either a low-risk or a high-risk group. High-risk was assigned if the infant had experienced one or more of the following risk factors: 1) Apgar score at $5 \mathrm{~min}<3$,2) respiratory problems, or 3 ) persistent ductus arteriosus (Table 1). None of the infants had a ROP plus disease. Neonatal morbidity of the brain was assessed with serial neonatal ultrasound scans. Periventricular hemorrhages and echodensities were classified according to the methods of Levene (16) and Jongmans (17) (Table 1). Twelve healthy full-term infants, who participated as a reference group in the

Table 1. Early characteristics of preterm children assessed at 6 years of age

$\begin{array}{ll}\text { Gender female/male } & 24 / 28 \\ \text { Maternal education, below/above } 12 \text { years } & 24 / 28 \\ \text { Gestational age at birth (wk) } & 29 \mathrm{SD} \pm 2.9 \\ \text { Birthweight (g) } & 1158 \mathrm{SD} \pm 337 \\ \text { Low-risk and high-risk }(n) & 31 / 21 \\ \text { Neonatal respiratory problems }(n) \dagger & 33 \text { no problem, } 19 \text { with problems } \\ \text { Neonatal ultrasound scan of the brain }(n) \ddagger & 22 \text { normal, } 13 \text { minor abnormality, } 17 \text { no data } \\ \text { Quality of General Movements }(n) & 14 \text { normal, } 35 \text { mildly abnormal, } 1 \text { definitely abnormal, }{ }^{1} 2 \text { no data } \\ \text { Bayley (37) PDI at } 6 \text { and } 12 \text { mo (median, mean, SD) } & 85,86 \mathrm{SD} \pm 14 \text { and } 85,86 \mathrm{SD} \pm 15 \\ \text { Bayley MDI at } 6 \text { and } 12 \text { mo (median, mean, SD) } & 99,98 \mathrm{SD} \pm 9 \text { and } 99,97 \mathrm{SD} \pm 11 \\ \text { Bayley Behavioral score at } 6 \text { and } 12 \text { mo (median, mean, SD) } & 121,119 \mathrm{SD} \pm 9 \text { and } 125,124 \mathrm{SD} \pm 7\end{array}$

$\dagger$ Respiratory problems included at least one of the following: a) respiratory illness needing surfactant treatment, b) $>1$ week of mechanical ventilation, c) pneumothorax.

$\ddagger$ Minor abnormalities on the neonatal ultrasound scan: peri/intraventricular haemorrhage grade 1 or 2, periventricular echodensities lasting for at least 14 days, ventriculomegaly (16,17). PDI, Psychomotor Developmental Index; MDI, Mental Developmental Index.

${ }^{1}$ In the analyses the single infant with definitely abnormal GMs was pooled with the infants with mildly abnormal GMs into the group "mildly abnormal GMs." 
early studies, also participated in the present follow-up study. The full-term infants had been born at Ullevaal Hospital, Oslo, with an appropriate birthweight (3878 $\pm 442 \mathrm{~g})$ and an Apgar score of at least 9 at $5 \mathrm{~min}(15)$.

Follow-up around the age of $6 \mathrm{y}$ was achieved in 52 of the 74 children (70\%). Loss to follow-up $(n=22)$ was due to migration $(n=12)$, time constraints of parents who stated that their children were doing well $(n=3)$, and inability to get in touch with the family ( $n=7$, two pairs of twins). The children lost to follow-up were no different in neonatal characteristics, quality of GM, or Bayley scores during infancy from the children included in the present project. Mean age of the preterm children was 74 mo SD \pm 7 chronological age. Mean age of the full-term children was 69 mo $(\mathrm{SD} \pm 1)$. Details on early characteristics of the preterm children included in the follow-up are summarized in Table 1. The Ethics Committee of region 1 of Norway approved the study, and all parents signed informed consent before participation.

Procedures and analysis. At the corrected ages of 4 and 6 mo, the kinematics of reaching and the kinetics of COP in supine position were recorded. The infants were presented with a toy to reach for above them at chest level, reflective markers were attached to the hands and the toy, and reaching was recorded with either a MacReflex or Peak Motus system. Goal-directed reaching ending in contact with the toy was defined as "successful." An Amti or Kistler forceplate placed below the lying infant recorded the displacement of the COP (14). The data from the two systems were processed with the same software. The analysis of the reaching kinematics focused on the number of MU derived from the velocity profile (13), the length and duration of the first MU relative to total movement path/time, and maximum hand velocity during each reach. In addition to these quantitative reaching parameters, a compound variable called reaching quality was used. Based on the performance of the full-term infants, reaching quality at 4 mo was considered as optimal when movements had a medium velocity (301-800 $\mathrm{m} / \mathrm{s}$ ), irrespective of the number of MU. At $6 \mathrm{mo}$, the criteria for optimal reaching were a medium velocity combined with the presence of $<3.5 \mathrm{MU}$ (13). The analysis of forceplate data focused on: a) the total-body COP displacement, defined as the distance travelled by the COP in medial-lateral and cranial-caudal directions during the reaching period; b) the $\mathrm{V}_{\max }$ of the $\mathrm{COP}$ displacement, determined from the derivative of the displacement curve; and, c) the number of directional changes, i.e. oscillations, in the COP displacement (14).

At 4 mo corrected age, the quality of GM was assessed from a video recording (3-5 $\mathrm{min}$ ) of spontaneous motor behavior in the preterm infants. Care was taken that the infant was in an active, noncrying behavioral state. One of the authors (M.H.A.), who was not apprised of the infants' clinical status, reaching and COP behavior, and follow-up data, classified the quality of GM into normal, mildly abnormal, or definitively abnormal (5). GM was relatively difficult (but not impossible) to assess in 18 infants due to the recurrent occurrence of spontaneous goal-directed behavior, such as mutual manipulation of hands or manipulation of clothes. This intermittent spontaneous goaldirected behavior was observed in particular in the low-risk preterm group; an observation that concurs with our previous finding of an accelerated maturation of goal-directed reaching in these infants (13).

Assessments at $6 y$ (chronological age). At follow-up, the children had two sets of neuromotor assessments. Both assessments were videotaped for later analysis. First, the Touwen neurologic examination (18) was carried out by one of the authors (B.F.). This examination focuses on the presence of MND. The signs of MND were grouped into functional clusters: mild abnormalities in muscle tone regulation, mildly abnormal reflexes, choreiform dyskinesia, mild coordination problems, mild problems in fine manipulative ability, and a group of miscellaneous dysfunctions, such as an excess of associated movements (19). On the basis of this examination, the children were grouped into four categories (19): a) neurologically normal, b) simple MND: dysfunction in two functional clusters, c) complex MND: dysfunction in more than two clusters, and d) CP. The classification CP implies the presence of a "classical" configuration of neurologic signs, such as the combination of a stereotyped posture and motility of the legs, an increased muscle tone and brisk tendon reflexes in the legs, and Babinski signs. Children with complex MND do not exhibit such classical combinations of signs. The functional difference between the three forms of neurologic dysfunction is that simple MND denotes impairment only and complex MND indicates functional impairment usually resulting in some degree of disability and social limitation, whereas $\mathrm{CP}$ is associated with disability and/or social limitation. M.H.A., who was not appraised of the children's early data, re-assessed the neurologic condition of the children on the basis of the video recordings. The few discrepancies in classification between B.F. and M.H.A. were discussed until consensus was reached. Second, motor function was assessed by means of the Movement ABC (20), which addresses hand motor skills (three items), ball skills (two items), and balance skills (three items). The items are scored $0-5,0$ being the optimal score. Total raw scores are presented in percentiles. Video recordings of Movement $\mathrm{ABC}$ were scored by one of the authors (E.H.), who was not appraised of the earlier data and the results of the Touwen examination.

The parents filled in two questionnaires on the behavior of their child: 1) the Checklist of the Movement $\mathrm{ABC}$, which provides information on motor abilities and play skills (20), and 2) the Achenbach Child Behavior Checklist $4-18$ y (CBCL) (21). The processing of the CBCL data were performed with the Assessment Data Manager Software version 1.1, 1991. The behavioral scores were categorized into internalizing and externalizing problems based on the subgroups such as withdrawl, somatic complaints, anxious/depressed, social problems or attention problems, and aggressive behavior. The competence scores were not provided because the majority of the children were about to start or had just started school.

Statistics. The nonparametric $\chi^{2}$ or Fisher exact tests were used to evaluate the association between group membership (full-term, preterm, low-risk, highrisk), dichotomized neonatal parameters (see definition in Table 1, in addition quality of GM), and the qualitative reaching parameter on the one hand and neurologic outcome on the other hand. The nonparametric Mann-Whitney test was used to assess associations between the continuous neonatal variables (birthweight and gestational age) and neurologic outcome, between group membership and dichotomized neonatal parameters on the one hand and scores on the Movement $\mathrm{ABC}$ on the other hand, between quantitative kinematics of reaching and COP data on the one hand and the neurologic outcome on the other hand. Finally, Spearman rank correlation was performed to evaluate the relationships between birthweight, gestational age, quantitative reaching data, COP data, and scores on the Movement ABC, and also between the quantitative reaching kinematics, the COP data, and scores on the checklists of Movement $\mathrm{ABC}$ and CBCL. In the majority of analyses, the data on the preterm and full-term children were pooled. When data on preterm children were analyzed separately, this is mentioned in the presentation of the results. The statistics were performed with SPSS version 11 (SPSS Inc., Chicago, IL). Differences with $p$ values $<0.05$ were considered to be statistically significant (two tailed). For the correlations, the level of significance was set at 0.01 to avoid the risk of making a type I error.

\section{RESULTS}

Neonatal data and developmental outcome. All the fullterm children were in a normal neurologic condition at $6 \mathrm{y}$. This outcome differed from that for the preterm children, who were more frequently classified as simple or complex MND ( $p$ $=0.003)$. This was true for both high-risk and low-risk children (LR $p=0.02$, HR $p=0.002$, Fig. 1a). The high-risk preterm children were more frequently classified as complex MND than the low-risk preterm children $(p=0.05)$ and the full-term children $(p=0.03$, Fig. 1a). With respect to the specific clusters of MND, we found that deviations in muscle tone were more frequently present in high-risk preterm children than in low-risk $(p=0.03)$ and full-term children $(p<$ 0.05 ). In addition, coordination problems were more often found in both low-risk and high-risk preterm infants than full-term infants ( $\mathrm{LR} p=0.04, \mathrm{HR} p=0.03$ ). The three groups did not differ significantly in fine manipulative ability, reflexes, or choreiform dyskinesia.

The 11 full-term children who completed the Movement $\mathrm{ABC}$ test performed normally, i.e. they scored above the 15th percentile. This performance differed from that of the preterm children, particularly from that of the high-risk preterm children, who more frequently scored below the 15 th percentile (Fig. 1b). One full-term child could not be scored because the child refused to do one item. In addition, the percentage of children with scores below the 5th percentile on the Movement $\mathrm{ABC}$ was higher for the high-risk children than for the low-risk children $(p=0.03$, Fig. $1 b)$. Both groups of preterm children scored significantly worse on the movement $\mathrm{ABC}$ total score and the subscore of hand motor skills than the full-term children (Table 2). There was a tendency for poorer subscores 

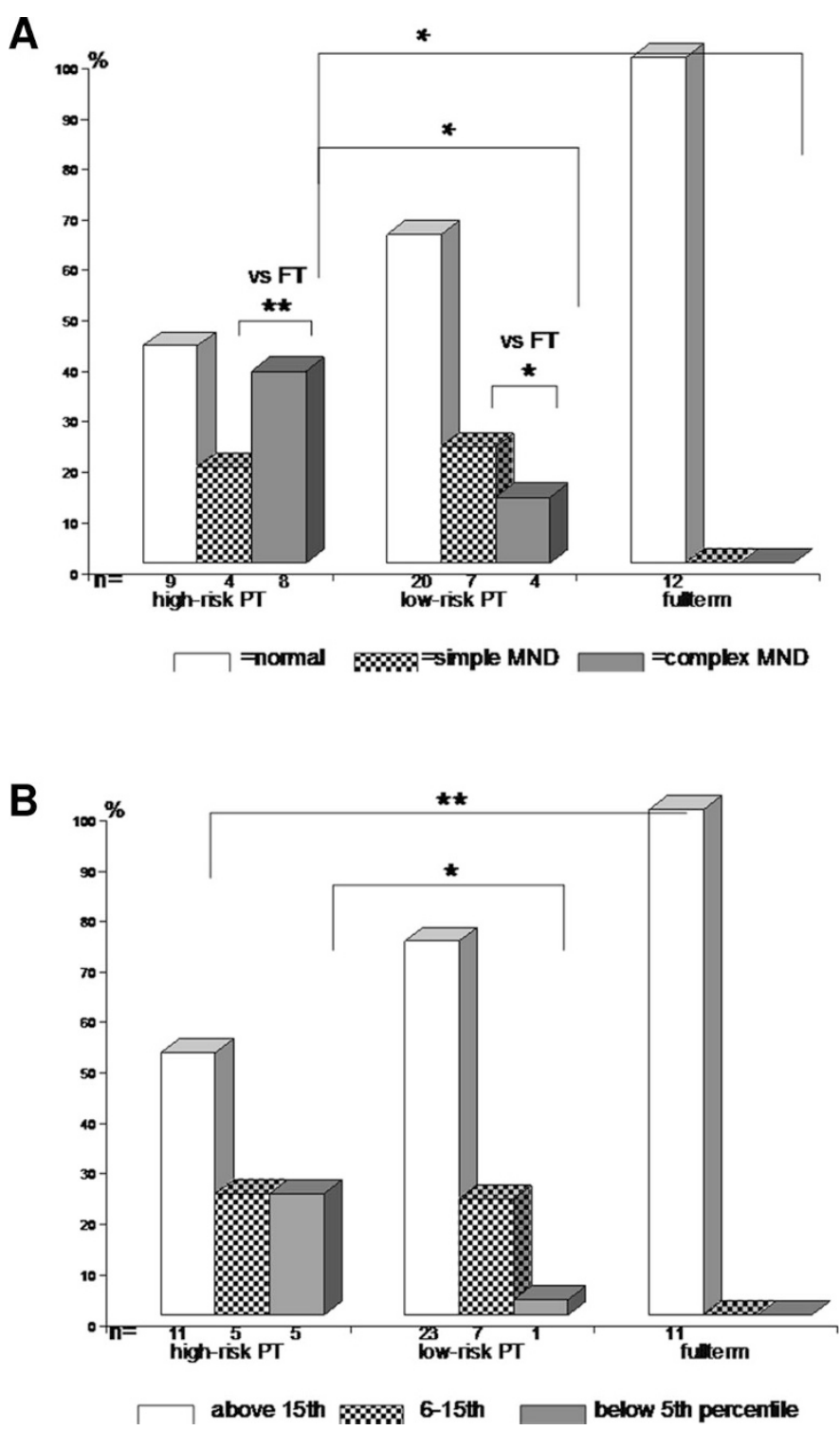

Figure 1. Distribution of neurologic condition $(a)$ and performance on the Movement $\mathrm{ABC}(b)$ at $6 \mathrm{y}$ in the three study groups: high-risk PT, low-risk PT, and full-term. Differences between groups: $* p<0.05$, $* * p<0.01$.

on balance skills in the preterm children compared with the full-term children ( $p=0.05$, Table 2$)$, although the groups did not differ in ball skills. The parents of preterm children tended to rate their children better on the Movement $\mathrm{ABC}$ Checklist than the parents of the full-term children, despite the fact that their children scored significantly worse on the Movement ABC. In fact, the parents of high-risk children rated their children the best (median: HR, 33; LR, 35; FT, 41.5; NS).

The differences in CBCL behavior were rather small. In general, the preterm children rated higher than the full-term children. This difference reached statistical significance for the score relating to internalizing problems (median: FT, 40; PT, 46; $p<0.04$ ) mainly due to a difference in anxious/depressed behavior (median: $\mathrm{FT}=0, \mathrm{PT}=1.5, p=0.02$ ).

Within the group of preterm infants, gestational age, birthweight, the presence of respiratory problems, and minor abnormalities on the neonatal ultrasound scan of the brain were not significantly related to later neurologic outcome. Gestational age, birthweight, and minor abnormalities on the ultrasound scan of the brain were also not related to performance on the Movement ABC. But the presence of respiratory problems was associated with worse scores on the Movement $A B C$ (median: problems $=8.5$ versus no problems $=7, p=0.004$ ). None of the neonatal characteristics were associated with CBCL behavior. Neither were the outcome variables related to age at follow-up or social class as indicated by level of maternal education.

Early reaching behavior and outcome. Information on reaching behavior was present for 38 preterms and 12 fullterms at 4 mo and 31 preterms and 11 full-terms at 6 mo. Within the preterm group the inability to reach successfully was related to the development of complex MND: out of the 7 children who could not reach, 5 developed complex MND, whereas of the 31 children who did reach, only 5 developed complex MND $(p=0.008$, Fig $2 a)$. Such a relationship was absent in the full-term children; 3 out of 12 infants did not reach successfully at $4 \mathrm{mo}$, but none of the children had complex MND. The odds ratio for developing complex MND in preterm infants unable to reach at 4 mo was 13 [confidence interval (CI) 95\%, 1.95-86.8]. At the age of 6 mo, one preterm child was unable to reach and he developed complex MND (Fig 2a).

The quantitative kinematic reaching variables at 4 and 6 mo were minimally related to the neurologic condition at school age, the exception to the rule being a relationship between a lower $\mathrm{V}_{\max }$ during reaching at 4 mo and increased trunk stiffness at school age $(p=0.009)$. Also, the quality of reaching at 4 mo showed hardly any relation to the neurologic condition at follow-up, the only trend we found was a weak association between nonoptimal reaching and coordination problems at school age $(p=0.06)$. However, the quality of reaching at 6 mo was significantly related to the development of complex MND ( $p=0.009$; odds ratio 13, CI 95\%, 1.4126.3) and the specific cluster of fine manipulative disability ( $p$ $=0.006$ ).

None of the reaching variables at 4 and 6 mo were related to scores on the Movement ABC (Fig. 2b), and only one to CBCL scores at $6 \mathrm{y}$ : a higher $\mathrm{V}_{\max }$ during reaching at 4 mo was associated with a worse attention (rho $=0.54, p=0.001$ ).

Early COP behavior and outcome. Information on COP behavior was present for 21 preterms and 8 full-terms at 4 mo and 23 preterms and 10 full-terms at $6 \mathrm{mo}$. None of the COP variables were related to the development of complex MND. However, a lower $\mathrm{V}_{\max }$ of COP in the medial-lateral direction at 4 mo was related to the presence of simple or complex MND $(p=0.04)$, and to the specific cluster of coordination problems $(p=0.04)$. The displacement of COP at 4 mo was not related to neurologic condition. At 6 mo no relation between COP behavior and neurologic outcome was found.

Performance on the movement $\mathrm{ABC}$ below the 15th percentile was associated with lower $\mathrm{V}_{\max }$ of $\mathrm{COP}$ in the mediallateral direction $(p=0.02)$ and a lower number of oscillations in the cranial-caudal direction $(p=0.02)$ at 4 mo, and with a smaller COP displacement in the medial-lateral direction ( $p=$ 0.04 ) and a lower number of oscillations in the cranial-caudal 
Table 2. Group allocation and motor assessment at school age

\begin{tabular}{lllll}
\hline & All preterm $n=52$ & High-risk PT $n=21$ & Low-risk PT $n=31$ & Full term $n=12$ \\
\hline Movement ABC & & & & \\
Total score median, (percentile 5-95) & $7.5(0.33-20.4)$ & $9.5(0.5-31.6)$ & $7(0.3-14.5)$ & $4(0-7.5)^{* *+\dagger \dagger+\ddagger}$ \\
Handmotor skills, median, (percentile 5-95) & $1.5(0-8.75)$ & $2(0-12)$ & $1(0-6)$ & $0(0-5)^{*}+\neq$ \\
Ball skills, median, (percentile 5-95) & $2.25(0-9)$ & $1(0-9.9)$ & $2.5(0-7.4)$ & $0.5(0-6)$ \\
Balance skills, median (percentile 5-95) & $1.75(0-11.2)$ & $1.5(0-11.5)$ & $2(0-9.1)$ & $0.25(0-5.5)^{*}$ \\
\hline
\end{tabular}

Full-term vs. preterm (PT): $* p<0.05, * * p<0.01$, full-term vs. low-risk PT: $\dagger p<0.05, \dagger \dagger p<0.01$, full-term vs. high-risk PT: $\ddagger p<0.05, \ddagger \ddagger p<0.01$.
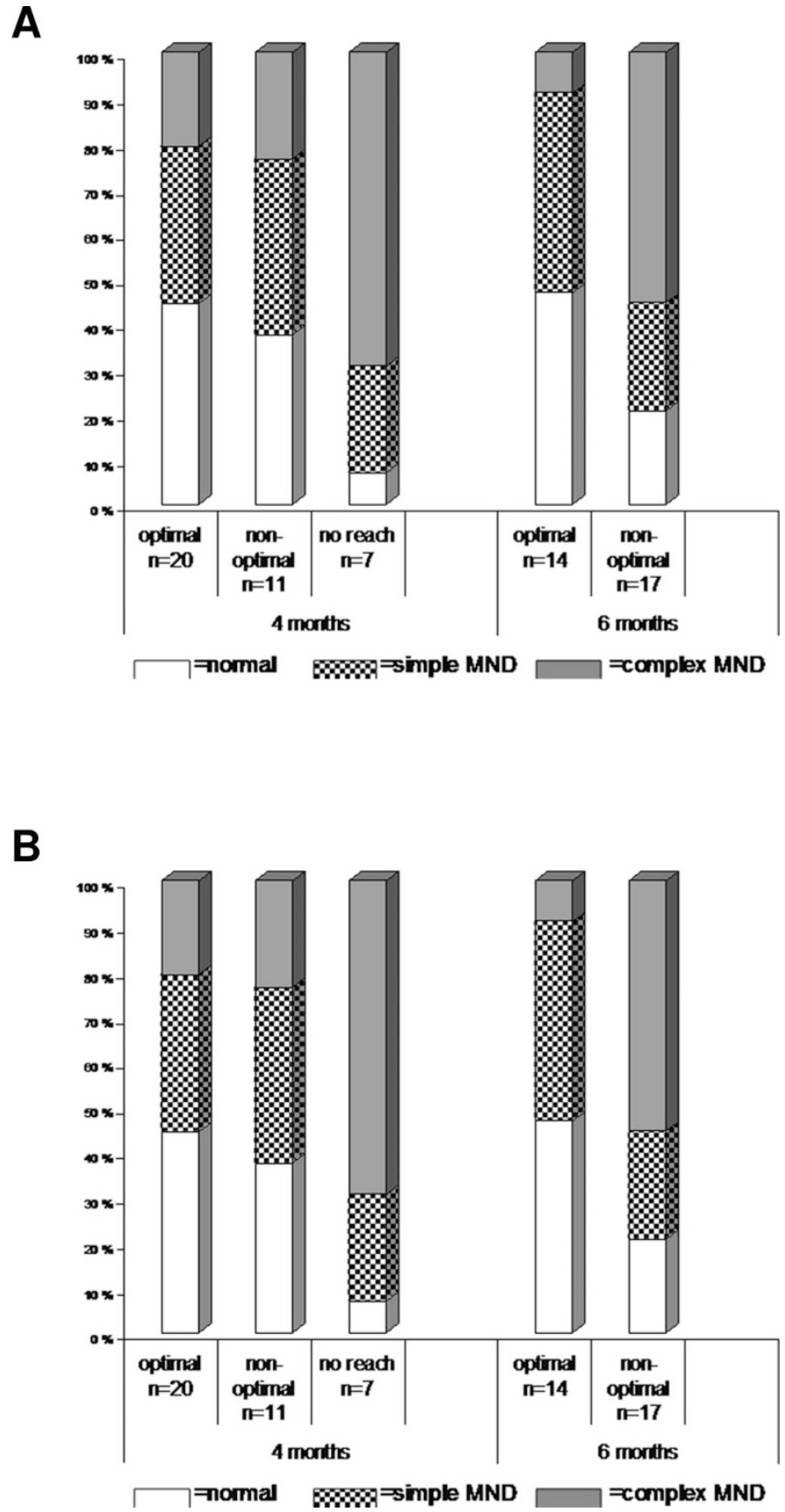

Figure 2. Quality of reaching in preterm children at 4 and 6 mo and neurologic condition (a) and performance on the Movement $\mathrm{ABC}(b)$ at $6 \mathrm{y}$. One infant of 6 mo was unable to reach; his data are added to the group with nonoptimal reaching behavior.

direction $(p=0.02)$ at $6 \mathrm{mo}$. The total score on the Movement $\mathrm{ABC}$ was not related to COP behavior at $4 \mathrm{mo}$, but it was related to a smaller COP displacement (rho $=-0.47, p=$
0.007 ) and a lower velocity (rho $=-0.45, p=0.01$ ) in the medial-lateral direction at 6 mo. Note that in Movement ABC a higher score indicates poorer performance. A higher balance subscore was related to a smaller COP displacement in the cranial-caudal direction (rho $=-0.49, p=0.006)$ and a lower $\mathrm{V}_{\max }$ in the medial-lateral direction ( $\mathrm{rho}=-0.51, p=0.005$ ) at $4 \mathrm{mo}$, but the subscores of hand motor and ball skills were not related to COP behavior.

$\mathrm{COP}$ behavior at $4 \mathrm{mo}$ was not related to behavior at $6 \mathrm{y}$ as scored on CBCL, but COP behavior at 6 mo was. A smaller COP displacement in the cranial-caudal direction was correlated with a higher rating on the internalizing problem scale (rho $=-0.50, p=0.006$ ). This internalizing behavior was apparent in the relationship between a smaller displacement in the medial-lateral direction and more anxious/depressed behavior (rho $=-0.49, p=0.007$ ) and more social problems (rho $=-0.51, p=0.005)$, and between a smaller displacement in the cranial-caudal direction and more somatic complaints (rho $=-0.47, p=0.01$ ).

Quality of GM and outcome. Of the 14 infants with normal $\mathrm{GM}$ at $4 \mathrm{mo}, 10$ were classified as neurologically normal, 2 as simple MND, and 2 as complex MND at school age, whereas of the 36 with abnormal GM, 17 had a normal outcome, 9 were classified as simple MND, and 10 as complex MND. The differences between the groups were, however, not statistically significant. Quality of GM was related in a similar way to the performance of the Movement ABC: of the 13 infants with normal GM, 11 scored above the 15 th percentile and 3 scored between the 5 th and 15 th percentiles. Of the 36 with abnormal GM, 21 scored above the 15 th percentile, 9 between the 5 th and 15 th percentile, and 6 below the 5th percentile (NS). Nevertheless, abnormal GM were related to a worse total score on the Movement $\mathrm{ABC}(p=0.03)$ and worse performance on the subcategory of ball skills $(p=0.01)$. The quality of GM were not related to scores on the CBCL.

\section{DISCUSSION}

The present study demonstrates that in preterm infants who do not develop CP, a nonoptimal quality of reaching at 6 mo and reduced postural behavior at 4 and 6 mo is associated with a less favorable neuromotor outcome at $6 \mathrm{y}$.

Before discussing the clinical and pathophysiological significance of our findings, the strengths and weaknesses of the study should be addressed. The study included a relatively small number of children, so caution is warranted in the interpretation of the results. Indeed, more studies are needed before the findings can be extrapolated to the general population. Our follow-up was hampered by $30 \%$ attrition. Fortu- 
nately, with regard to neonatal and early developmental data, the loss to follow-up was nonselective. The strength of the study is that it provides novel and valuable information on the detailed quantitative and qualitative aspects of neuromotor behavior in young preterm infants and relates these findings to later childhood development. This information may direct assessment during infancy and help to program better aimed intervention in an effort to optimize neuromotor development of preterm infants in the future.

Interestingly, we found that preterm infants who were not able to reach successfully at 4 mo were at high risk for the development of complex MND. There was no such relationship in the full-term group, which is in agreement with the data presented by others, indicating that successful reaching in full-term infants develops between 4 and 5 mo (22). The finding that in preterm infants the inability to reach successfully at 4 mo is related to an adverse developmental outcome suggests that preterm infants with brain dysfunction may be unable to profit from the additional extrauterine experience. In preterms without noteworthy brain dysfunction, this induces an acceleration of the development of reaching and visual abilities $(13,23)$. In addition, a nonoptimal quality of reaching at 6 mo was associated with the development of complex MND, and in particular with fine manipulative disability. Hadders-Algra and co-workers $(5,6)$ reported that abnormal GM at $2-4$ mo are also related to complex MND and fine manipulative dysfunction. Both nonoptimal reaching and abnormal GM may point to a deficit in the planning or coordination of movements-in reaching, an inadequate number of MU (24) and inadequate velocity profile; in GM, a deficiency in movement complexity and variation (25), an inability to fine-tune motor output, and nonfluent movements (26). It is plausible that a deficiency in planning or coordinating movements puts the child at risk for complex MND and fine manipulative disability. The fact that we could not fully replicate the findings of Hadders-Algra et al. $(5,6)$ may be attributed to the relatively low number of infants with normal GMs in the present study.

Reaching toward a specific location in space demands accuracy of the movement of the hand, whereas the ability to modify the posture to the task is one of the rate-limiting factors for successful action (27). We did not consider the reduced postural motility at early age as rate limiting for reaching because it was associated with a good quality of reaching. Instead, we surmised that this behavior was an adequate functional adaptation to extrauterine demands (14). However, in the normal infant, an ongoing modulation and exploration of solutions to acquire successful reaching and grasping in different situations is present $(28,29)$. It has been reported that preterm infants lack this capacity to modulate and show excessive trunk muscle activity while reaching (30), behavior that might be analogous to the reduced postural motility we observed, and the postural behavior that has been observed during various spontaneous motor activities by others $(10,31)$. The present data indicated that the relatively immobile postural behavior was related to a less favorable neurologic outcome such as simple MND, and the specific cluster of coordination problems, poor performance on the movement $\mathrm{ABC}$ and balance skills, but not to complex MND. Simple MND, which is considered to reflect normal but nonoptimal brain function (19), may be related to stress during early life, such as preterm birth (32). In animals, stress during early life may induce permanent alterations in the structure and function of the CNS, in particular in the monoaminergic systems (33). Prenatal stress in primates may also result in minor dysfunctions in balance and behavioral problems (34). We also found that the relatively immobile postural behavior was linked to some extent to behavioral problems, i.e. to internalizing behavior. The internalizing behavior may be related to the experience of awkwardness and failure in motor skills (35), but it could also be related to dysfunctions in the monoaminergic systems, especially in the serotonergic system (36). An additional or alternative explanation for the association between reduced postural behavior at an early age and nonoptimal motor outcome might be that both phenomena might be caused by an altered muscle development in preterm infants (8).

\section{CONCLUSION}

The present study indicated that in preterm infants who do not develop $\mathrm{CP}$ a lack of successful reaching at 4 mo and an inadequate quality of reaching at 6 mo (corrected age) are sensitive markers of clinically significant forms of brain dysfunction, such as complex MND. The presence of these signs suggests that the infant should be monitored carefully and indicates that the infant is in need of therapeutic guidance. A relatively immobile postural behavior has less clinical significance. Nevertheless, it is related to less optimal motor and behavioral development. Thus, when aiming for optimal developmental outcome, we may consider intervention that stimulates the infant to explore more motor solutions.

\section{REFERENCES}

1. de Vries AM, de Groot L 2002 Transient dystonias revisited: a comparative study of preterm and term children at 21/2 years of age. Dev Med Child Neurol 44:415-421

2. de Groot L 2000 Posture and motility in preterm infants. Dev Med Child Neurol 42:65-68

3. Drillien CM, Thomson AJ, Burgoyne K 1980 Low-birthweight children at early school-age: a longitudinal study. Dev Med Child Neurol 22:26-47

4. de Groot L, Hopkins B, Touwen B 1995 Muscle power, sitting unsupported and trunk rotation in pre-term infants. Early Hum Dev 43:37-46

5. Sommerfelt K, Pedersen P, Ellertsen B, Markestad T 1996 Transient dystonia in non-handicapped low-birthweight infants and later neurodevelopment. Acta Paediatr 85:1445-1449

6. Samson JF, de Groot L, Bezemer PD, Lafeber HN, Fetter WP 2002 Muscle power development during the first year of life predicts neuromotor behaviour at 7 years in preterm born high-risk infants. Early Hum Dev 68:103-118

7. Fallang B, Saugstad OD, Grøgaard J, Hadders-Algra M 2003 Kinematic quality of reaching movements in preterm infants. Pediatr Res 53:836-842

8. Fallang B, Saugstad OD, Hadders-Algra M 2003 Postural adjustments in preterm infants at 4 and 6 months post-term during voluntary reaching in supine position. Pediatr Res 54:826-833

9. Fallang B, Saugstad OD, Hadders-Algra M 2000 Goal directed reaching and postural control in supine position in healthy infants. Behav Brain Res 115:9-18

10. Levene MI, Fawer CL, Lamont RF 1982 Risk factors in the development of intraventricular haemorrhage in the preterm neonate. Arch Dis Child 57:410-417

11. Jongmans M, Henderson S, De Vries L, Dubowitz L 1993 Duration of periventricular densities in preterm infants and neurological outcome at six years of age. Arch Dis Child 69:9-13

12. Touwen BC 1979 Examination of the Child with Minor Neurological Dysfunction, 2nd Ed. William Heinemann Medical Books, London

13. Hadders-Algra M 2003 Developmental coordination disorder: is clumsy motor behaviour caused by a lesion of the brain at early age? Neural Plast 10:39-50

14. Henderson SE, Sugden DA1992 Movement Assessment Battery for Children. The Psychological Corporation, London

15. Achenbach TM 1991 Manual for the Child Behavior Checklist/ 4-18 and 1991 Profile. University of Vermont, Department of Psychiatry, Burlington, VT 
16. Von Hofsten C 1984 Developmental changes in the organization of prereaching movements. Dev Psychol 20:378-388

17. Van Hof-van Duin J, Heersema DJ, Groenendaal F, Baerts W, Fetter WP 1992 Visua field and grating acuity development in low-risk preterm infants during the first $21 / 2$ years after term. Behav Brain Res 49:115-122

18. Berthier NE 1996 Learning to reach: a mathematical model. Dev Psychol 32:811-823

19. Hadders-Algra M 2000 The neuronal group selection theory: a framework to explain variation in normal motor development. Dev Med Child Neurol 42:566-572

20. Hadders-Algra M, Klip-Van den Nieuwendijk AW, Martijn A, van Eykern LA 1997 Assessment of general movements: towards a better understanding of a sensitive method to evaluate brain function in young infants. Dev Med Child Neurol 39:89-99

21. Hopkins B 2003 Development of action and the clinical continuum. Neural Plast $10: 15-25$

22. Thelen E, Corbetta D, Kamm K, Spencer JP, Schneider K, Zernicke RF 1993 The transition to reaching: mapping intention and intrinsic dynamics. Child Dev 64:1058 1098

23. van der Fits IB, Klip AW, van Eykern LA, Hadders-Algra M 1999 Postural adjustments during spontaneous and goal-directed arm movements in the first half year of life. Behav Brain Res 106:75-90
24. van der Fits IB, Flikweert ER, Stremmelaar EF, Martijn A, Hadders-Algra M 1999 Development of postural adjustments during reaching in preterm infants. Pediatr Res $46: 1-7$

25. Gorga D, Stern FM, Ross G, Nagler W 1988 Neuromotor development of preterm and full-term infants. Early Hum Dev 18:137-149

26. Hellerud BC, Storm H 2002 Skin conductance and behaviour during sensory stimulation of preterm and term infants. Early Hum Dev 70:35-46

27. Weinstock M 2001 Alterations induced by gestational stress in brain morphology and behaviour of the offspring. Prog Neurobiol 65:427-451

28. Schneider ML, Coe CL 1993 Repeated social stress during pregnancy impairs neuromotor development in the primate infant. J Dev Behav Pediatr 14:81-87

29. Dewey D, Kaplan BJ, Crawford SG, Wilson BN 2002 Developmental coordination disorder: associated problems in attention, learning, and psychosocial adjustment. Hum Mov Sci 21:905-918

30. Ryan ND, Varma D 1998 Child and adolescent mood disorders-experience with serotonin-based therapies. Biol Psychiatry 44:336-340

31. Bayley Scales of Infant Development 1993 Manual, 2nd Ed. The Psychological Corporation, Harcourt Brace \& Company, San Antonio, TX 